\title{
Fracture prevention in osteoporosis: assessing risks, providing solutions
}

\author{
J.-Y. Reginster • M. L. Brandi
}

Received: 16 March 2010 / Accepted: 16 March 2010

(C) International Osteoporosis Foundation and National Osteoporosis Foundation 2010

Osteoporosis is the most common skeletal disorder in the elderly, being characterised by impaired bone mass and microarchitecture, bone strength and, consequently, increased risk of fracture. As the worldwide population ages, the population prevalence of osteoporosis is also increasing, and it is therefore particularly important to manage the disease which will affect more patients for longer. Currently, osteoporosis is defined using bone mineral density (BMD) thresholds determined by dual-energy X-ray absorptiometry; however, this definition does not entirely reflect the spectrum of severity of the disease that provides a variable increase in fracture risk. Many osteoporotic fractures do not come to clinical attention, and osteoporosis is still underdiagnosed. Whilst osteopenia is considered a lesser degree of bone loss than osteoporosis, it nevertheless can be of concern when it is associated with other risk factors for fracture.

In attempts to identify those individuals at a risk of fracture high enough to warrant pharmacotherapy, several algorithms have been developed, such as FRAX, that combine bone mineral density and other clinically identifiable risk factors to estimate a treatment-naïve individual's absolute fracture risk over a defined time interval. The

J.-Y. Reginster $(\bowtie)$

Department of Public Health Services, University of Liège, 45, quai G. Kurth, 4020 Liège, Belgium e-mail: jyreginster@ulg.ac.be

\section{L. Brandi $(\square)$}

Department of Internal Medicine, Metabolic Diseases Unit, University of Florence,

Viale G. Pieraccini, 6,

50139 Florence, Italy

e-mail: m.brandi@dmi.inifi.it effects of current or previous pharmacotherapy on these risk estimates are difficult to model. The aim of management of osteoporosis is the prevention of bone fractures by reducing bone loss or, preferably, by increasing bone density, improving bone microarchitecture and, consequently, bone strength. An ideal treatment would be efficient in reducing fracture irrespective of a patient's fracture history or identified baseline risk factors.

Until recently, there were two main therapeutic options available for the management of patients at high risk of osteoporotic fractures. The antiresorptive agents such as bisphosphonates and raloxifene that reduce bone resorption and the anabolic agents such as PTH and its derivatives that increase bone formation. Strontium ranelate is a novel osteoporosis medication in that it possesses both antiresorptive and anabolic properties. This dual mode of action rebalances bone turnover in favour of bone formation and respects bone as a living tissue. In clinical trials, antifracture efficacy has been proven at vertebral, nonvertebral, and hip sites.

This issue commences with a special guest article focusing on microarchitecture and the importance of advances in bone quality assessment. In an attempt to identify more patients at risk of osteoporosis, the issue follows up with an article outlining the background and development of the FRAX tool. The subsequent articles outline how to address the main risks identified in FRAX with strontium ranelate. These are age, disease severity, which includes BMD and previous fracture history, gender, glucocorticoid use, and lifestyle habits. It is important to note that physicians should recommend bone mineral density testing for younger women at risk and for postmenopausal women under 65 years who have risk factors for osteoporosis other than being postmenopausal, in order to identify more patients who may require treatment. The issue concludes with an important comparative analysis of different ways of evaluating treatments for 
osteoporosis. It is hoped that this will help clinicians in their own identification of patients at risk of osteoporosis and provide information regarding options for treatment.

Conflicts of interest J.-Y. Reginster (on behalf of the Department of Public Health, Epidemiology and Health Economics of the University of Liège, Liège, Belgium): consulting fees or paid advisory boards: Servier, Novartis, Negma, Lilly, Wyeth, Amgen, GlaxoSmithKline,
Roche, Merckle, Nycomed, NPS, Theramex, UCB; lecture fees when speaking at the invitation of a commercial sponsor: Merck Sharp and Dohme, Lilly, Rottapharm, IBSA, Genevrier, Novartis, Servier, Roche, GlaxoSmithKline, Teijin, Teva, Ebewee Pharma, Zodiac, Analis, Theramex, Nycomed, Novo-Nordisk; grant support from industry: Bristol Myers Squibb, Merck Sharp \& Dohme, Rottapharm, Teva, Lilly, Novartis, Roche, GlaxoSmithKline, Amgen, Servier.

M.L. Brandi is a consultant for and has received honoraria and grant/research support from MSD, Procter \& Gamble, Servier, Nycomed, Glaxo, NPS and Amgen. 